BNL- -48048

DE93 001439

\title{
Design Studies on the RHIC Position Monitor System
}

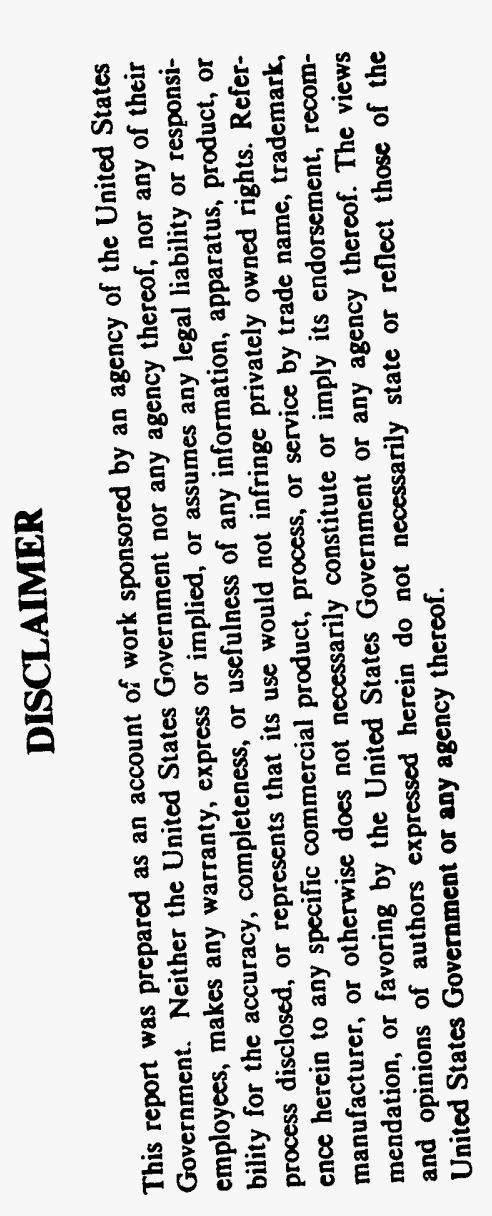

Tom Shea

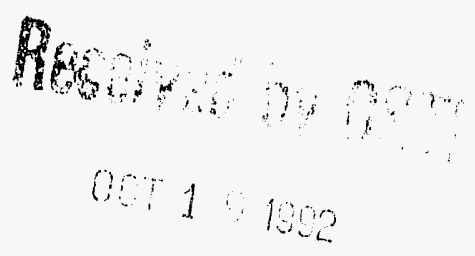

September 1992

\author{
R H I C P R O J E C T \\ Brookhaven National Laboratory \\ Associated Universities, Inc. \\ Upton, NY 11973
}




\title{
Design Studies on the RHIC Position Monitor System
}

\author{
Tom Shea
}

\subsection{Introduction}

The Relativistic Heavy Ion Collider (RHIC) under construction at Brookhaven National Laboratory will consist of two superconducting storage rings $3.8 \mathrm{~km}$ in circumference sharing one tunnel in a side by side configuration. After injection from the existing AGS, the collider will accelerate and store a range of ion species extending from protons through gold to energies of $250 \mathrm{GeV}$ for protons and $100 \mathrm{GeV} /$ nucleon for fully stripped gold ions. Storage times should exceed 10 hours. As in most modern colliders the RHIC beam position monitor system is the most extensive beam diagnostic in terms of both cost and channel multiplicity. The final system will consist of approximately 500 position monitors with over 600 measurement planes of electronics. Development of this system began early so that the monitors would be available for installation into the magnet cryostats. The following document summarizes some of the efforts undertaken during the design phase of this system. Because much of this work is still in progress, the document is necessarily incomplete and some details may not reflect the final system configuration.

\subsection{System Considerations}

\subsection{RHIC parameters}

A useful subset of the RHIC parameter list is shown in Table 1.

TABLE 1. Beam parameters

\begin{tabular}{lll}
\hline \multicolumn{1}{c}{ PARAMETER } & $\begin{array}{c}\text { NOM. } \\
\text { VALUE }\end{array}$ & \multicolumn{1}{c}{ COMMENTS } \\
\hline F'volution frequency & $78 \mathrm{kHz}$ & varies by <1\% \\
Bunching Frequency & $4.45 \mathrm{MHz}$ & during first operation \\
& $8.9 \mathrm{MHz}$ & possible upgrade with fast injection kicker \\
Charge in single bunch & $1 \times 10^{11} \mathrm{e}$ & for protons under nominal operating conditions \\
& $79 \times 10^{9} \mathrm{e}$ & for gold ions under nominal operating conditions \\
& $1 \times 10^{10} \mathrm{e}$ & pilot bunch during setup and commissioning \\
& $3 \times 10^{11} \mathrm{e}$ & possible upgrade \\
bunch length at injection & $17 \mathrm{~ns}$ & measured at base \\
bunch iength (min) & $4 \mathrm{~ns}$ & measured at base
\end{tabular}




\subsection{System specifications}

\subsubsection{Resolution}

In this context, the resolution is determined by the signal to noise ratio and short term (relative to time between calibrations) stability. The noise is dominated by thermal noise, digital noise due to digitizer quantization, and electromagnetic interference. Table 2 summarizes the resolution that we expect from a system consisting of the position monitors described in Section 3.1 and the electronics described in Section 4.2. Both analytical calculations and practical experience with other systems indicate that these resolutions are obtainable.

TABLE 2. Expected system resolution

\begin{tabular}{lll}
\hline \multicolumn{1}{c}{ MODE } & RESOLUTION & \multicolumn{1}{c}{ COMMENTS } \\
\hline Single bunch, first turn & $1 \mathrm{~mm}$ & $10^{10}$ protons per bunch \\
Single bunch, turn by turn & 0.1 & Full bunch intensity, $78 \mathrm{kHz}$ data rate \\
Closed orbit & $<<0.1$ & Averaged over many turns, 1 kHz data rate
\end{tabular}

\subsubsection{Accuracy}

In order to minimize the aperture requirements that arise from the emittance growth induced by intrabeam scattering, RHIC was designed with a rather strong focussing lattice. This has led to a strong chromaticity correction system that makes the machine sensitive to beam displacements relative to the sextupole axis. Therefore, a tolerance of $\pm 0.13 \mathrm{~mm}$ RMS was placed on the accuracy of closed orbit measurements relative to the sextupole. This requirement reflects the following considerations:

- the tolerance is small compared to the expected closed orbit distortions observed in a typical corrected lattice

- a beam displaced in the sextupole by this amount will sample random quadrupole errors comparable to the errors in the main quads

- this tolerance is technically achievable (although not with trivial effort)

The error will consist of two general components: mechanical alignment tolerance and uncalibrated electrical effects. As demonstrated in existing systems, the electrical effects can be minimized by a straight forward calibration. The more system specific mechanical tolerances are not yet well quantified. Efforts to minimize the two components are detailed in the following sections.

\subsection{The Position Monitor}

This section describes the mechanical design and predicted electrical performance of the position monitor pickup assembly that is currently in the prototyping stage. As we perform mechanical and electrical tests on the prototypes, the design will evolve into a production quality unit. In addition, several modified versions of this basic prototype design will be 
made to fulfill special requirements. The collection of monitors that will ultimately be required in the two collider rings is detailed in Table 3 . The apertures given in the table are actually the coil diameters of the adjacent magnets.

TABLE 3. Types of position monitors in the two collider rings

\begin{tabular}{|c|c|c|c|c|}
\hline & LOCATION & APERTURE (CM) & STYLE & NUMBER \\
\hline \multirow[t]{5}{*}{ Type 1} & Arc quads Q10-Q10 & 8 & cold-single plane & 276 \\
\hline & Q9 & 8 & cold-single plane & 24 \\
\hline & Q6 & 8 & cold-single plane & 24 \\
\hline & Q5 & 8 & cold-single plane & 24 \\
\hline & & & Subtotal & 348 \\
\hline \multirow[t]{3}{*}{ Type 2} & Q8 & 8 & cold-dual plane & 24 \\
\hline & Q7 & 8 & cold-dual plane & 24 \\
\hline & & & Subtotal & 48 \\
\hline \multirow[t]{3}{*}{ Type 3} & Q4 inboard & 13 & warm-dual plane & 24 \\
\hline & Q3 outboard & 13 & warm-dual plane & 24 \\
\hline & & & Subtotal & 48 \\
\hline Type 4 & DX inboard & 13 & $\begin{array}{l}\text { warm dual plane } \\
\text { (directional) }\end{array}$ & 12 \\
\hline \multirow[t]{3}{*}{ Type 5} & Q1 & 13 & cold-dual plane & 24 \\
\hline & & & Total monitors & 480 \\
\hline & & & Total channels & 636 \\
\hline
\end{tabular}

Several interesting variations on the basic position monitor design are apparent in the table. The Type 1 monitor is our current prototype and will be described in more detail in Section 3.1. On either side of the intersection regions a large aperture Type 4 monitor will be used to observe the position of the intersecting beams relative to the detector center. Because the beams from the two rings will be merged at these points, the directionality of these monitors will be utilized to separate the signals from each beam. The Type 4 are the only monitors in the collider that have this directional characteristic.

The choice of shorted electrodes for all monitors but the Type 4 deserves some discussion. For a relativistic beam, the signal at the electronics is identical for a back terminated stripline and a shorted stripline. Additionally, if care is taken to properly match the coaxial geometry to the stripline, high $Q$ resonances that could lead to a narrowband coupling impedance can be avoided. The failure mode in the case of an opened connection at the feedthrough is resonant, but the design of this connection should certainly be robusi 
enough to avoid this condition. The shorted electrode has several thermal advantages as well:

- a cold back termination that could dissipate reflected power at $4^{\circ} \mathrm{K}$ is avoided

- the alternative warm termination and the cable's attendant conductive thermal load is avoided

- differential thermal expansion between the stripline and the cavity is minimized thereby avoiding a source of stress on the cold feedthroughs

The shorted striplines should also be more economical than a directional coupler design.

\subsection{Mechanical design}

\subsubsection{The weldment}

A mechanical sketch of the Type 1 position monitor is shown in Figure 1. The striplines are approximately $20 \mathrm{~cm}$ long and each subtends an $80^{\circ}$ angle azimuthally. The mounting flange shown on the left will be bolted to a mating flange on the sextupole end of the corrector-quadrupole-sextupole (CQS) assembly. The large tapered shell forms the inner surface of the helium containment buffer volume.To protect the beam vacuum, an insulating vacuum shields the beam vacuum welds from the helium. In the current prototypes, most of these welds are electron beam welds, but the possibility of brazing the assembly is currently under investigation.

The tolerance of the electrical center to the mechanical center is expected to be about $0.15 \mathrm{~mm}$. I $\mathrm{f}$ this is the case for the production units, each monitor will have to be measured and the results of these measurements could be made available in the on line data base for correction. Alternatively, the striplines could be mechanically moved into position to avoid the bookkeeping requirements. A position monitor mapping facility has already been constructed and tested and will be used to investigate these techniques.

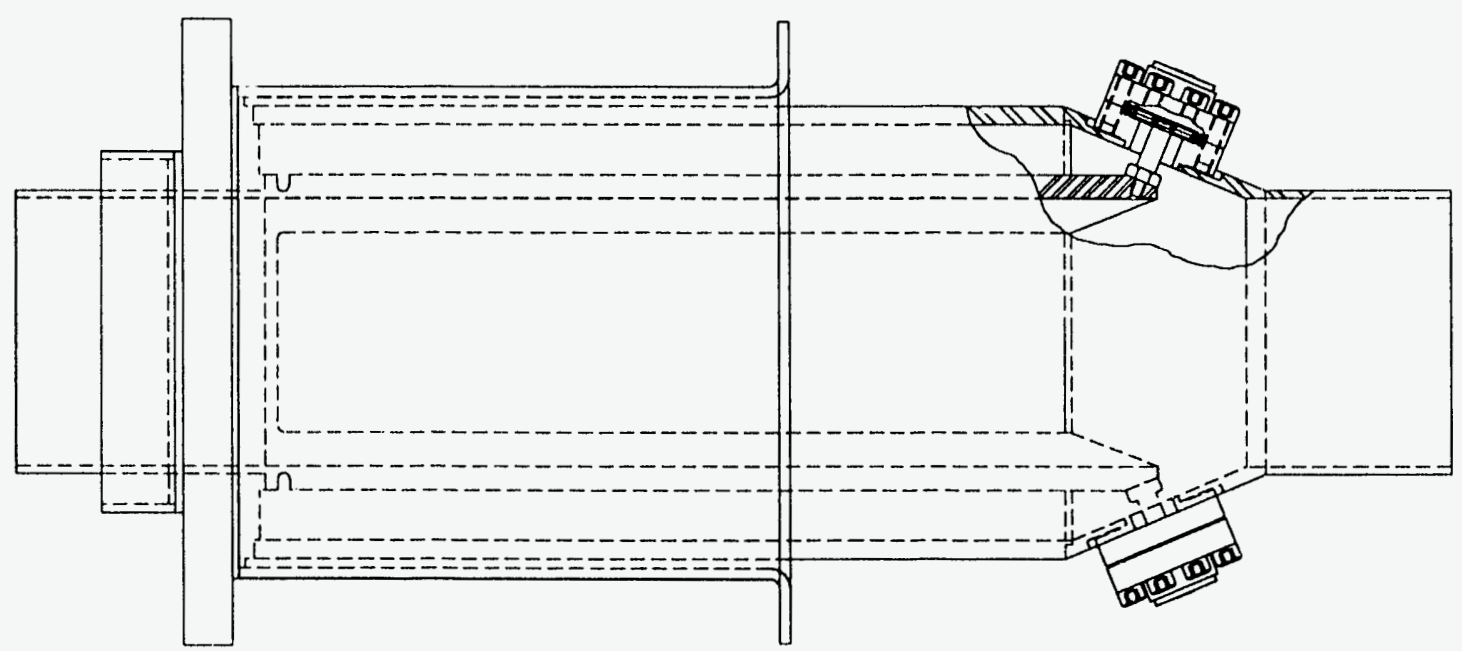

FIGURE 1. The prototype RHIC beam position monitor

Design Studies on the RHIC Position Monitor System Seprember 30, 1992 


\subsubsection{Feedthroughs}

The cold coaxial feedthroughs are a very important component from both electrical performance and reliability considerations. An alumina feedthrough is the conservative choice, but several higher performance glass-ceramic feedthroughs have been tested with excellent results. The simple construction of this newer technology could make them an economical alternative. The feedthroughs are removable by virtue of a conflat seal on the outer shell and a bellows contact on the inner conductor. This bellows contact is capable of absorbing the construction tolerances while imparting minimal stress on the feedthrough center pin.

\subsubsection{Cryogenic cables}

Semirigid coaxial cables will connect the cold feedthrough on the position monitor to the warm feedthrough on the outer cryostat shell. With a copper on stainless steel construction, the 0.141 " diameter cables should provide the necessary signal integrity with an acceptable heat load on the cryogenic system. A 5 foot long cable properly stationed to the $55^{\circ} \mathrm{K}$ heat shield should inflict a $139 \mathrm{~mW}$ load on the $4^{\circ} \mathrm{K}$ system and a $315 \mathrm{~mW}$ load on the $55^{\circ} \mathrm{K}$ system. The two radiation hard dielectrics being considered for this environment are $\mathrm{SiO}_{2}$ and $\mathrm{PEEK}$.

\subsection{Electrical considerations}

\subsubsection{Characteristic impedance}

The stripline geometry shown in Figure 1 is the result of several iterations of the cross section dimensions. The following prescription was used to calculate the characteristic impedance for each trial geometry:

1. As illustrated by the cross section in Figure 2, the finite clement program PE2D ${ }^{1}$ was used to model the position monitor's sum mode.

2. From the resulting fields, the stored energy per unit length was calculated.

3. From this stored energy, the capacitance per unit length, and therefore the characteristic impedance of the stripline in vacuum was calculated.

Measurements on a copper model agreed with the numerically calculated values to within the construction tolerances of the model. For example, one stripline measured $49 \Omega$ on the network analyzer when the predicted impedance was $50 \Omega$.

\subsubsection{Expected signals}

The signals expected at the position monitor ports are calculated as part of a full system simulation implemented in LabVIEW ${ }^{2}$. A screen shot of the panel for this simulation is

1. Vector Fields Inc. 


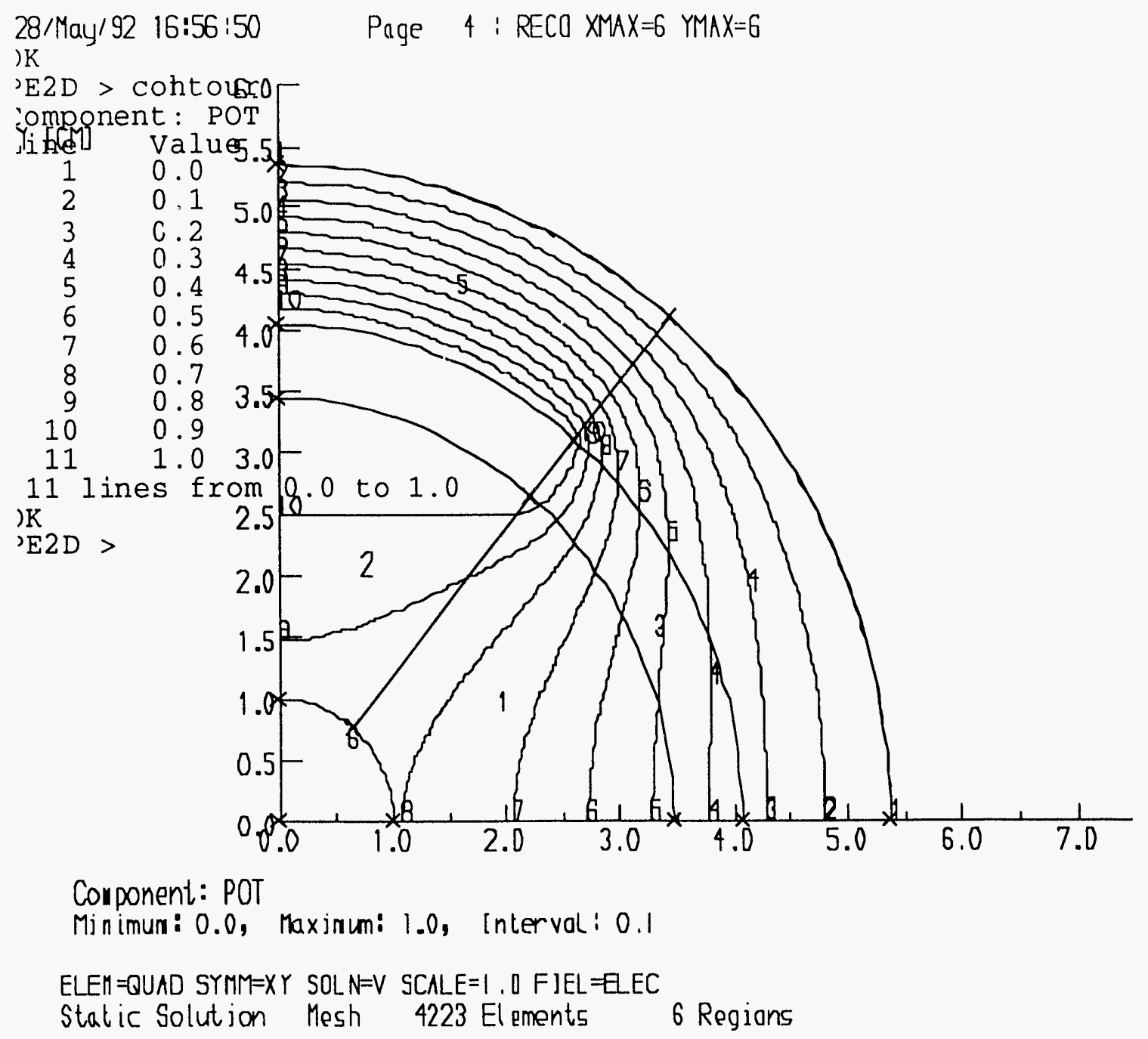

FIGURE 2. Sum mode of stripline

shown in Figure 3. This implementation allows engineers and technicians to adjust parameters in real time and "see" how a system might perform under various circumstances. For example, by looking at the peak voltages of the pulse doublet at the two operational extremes, we can obtain some idea of the dynamic range capability required of the position monitor receivers.

The minimum signal:

- small intensity pilot bunch $\left(10^{10}\right.$ protons per bunch)

- bunch at maximum displacement away from port of interest $(-20 \mathrm{~mm}$ off center)

- bunch length is maximum at injection (17 $\mathrm{ns}$ at base)

- leads to peak voltage of $21.2 \mathrm{mV}$

2. LabVIEW 2 for the Macintosh by National Instruments, Austin TX, USA

Design Studies on the RHIC Position Monitor System Septenther 30, 1992 


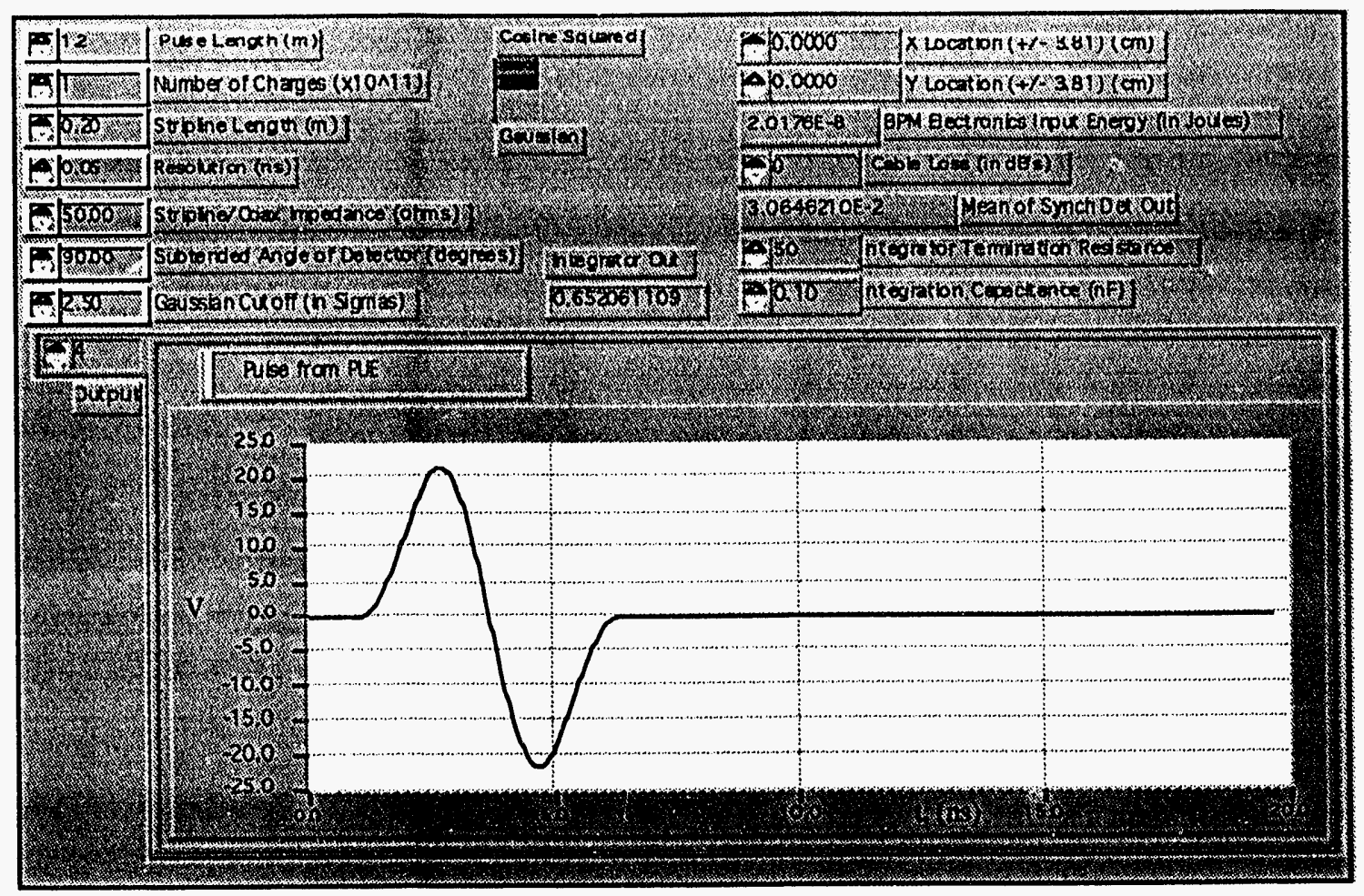

FIGURE 3. BPM simulation panel

And the maximum:

- $3 \times$ single bunch design intensity $\left(3 \times 10^{11}\right.$ protons per bunch)

- bunch at maximum displacement toward port of interest ( $+20 \mathrm{~mm}$ off center)

- minimum bunch length after transfer to storage RF system (4 $\mathrm{ns}$ at base)

- leads to a peak voltage of $68.6 \mathrm{~V}$

The peak voltages mentioned above suggest a dynamic range of about $70 \mathrm{~dB}$. Several effects are not considered in this simple analysis. For example by properly selecting the processing frequency range, the effect of bunch length on the dynamic range could be reduced. Furthermore, one would not expect a full upgrade-intensity beam to have its centroid located at the limit of the dynamic aperture. Amplitude variation due to different cable lengths must also be handled by the system.

\subsubsection{Linear aperture}

The linear aperture of a stripline position monitor was investigated with the following technique:

1. Assuming a filament beam and ignoring the edge effects at the stripline, we calculated the output power ratio at the position monitor ports for all positions within the monitor. These values define a function 


$$
\frac{P_{A}}{P_{B}}=g(x, y),
$$

where $\mathrm{x}$ and $\mathrm{y}$ are the cartesian coordinates of the filament location and $\mathrm{P}_{\mathrm{A}}$ and $\mathrm{P}_{\mathrm{B}}$ are the electrical powers at each port of the position monitor. The $\mathrm{x}$ axis is taken to be the axis of measurement.

2. Using the above assumptions we can also calculate the inverse function for a filament beam on the axis of meas'urement

$$
x=f\left(\left.\frac{P_{A}}{P_{B}}\right|_{y=0}\right)
$$

This defines the on-axis transfer function $f$.

3. When $\mathrm{y} \neq 0$, i.e. the beam is off-axis, we can still use the function $f$ defined in Equation 2 to calculate the beam position from the output signal power ratio:

$$
x_{\text {calc }}=f\left(\frac{P_{A}}{P_{B}}\right)
$$

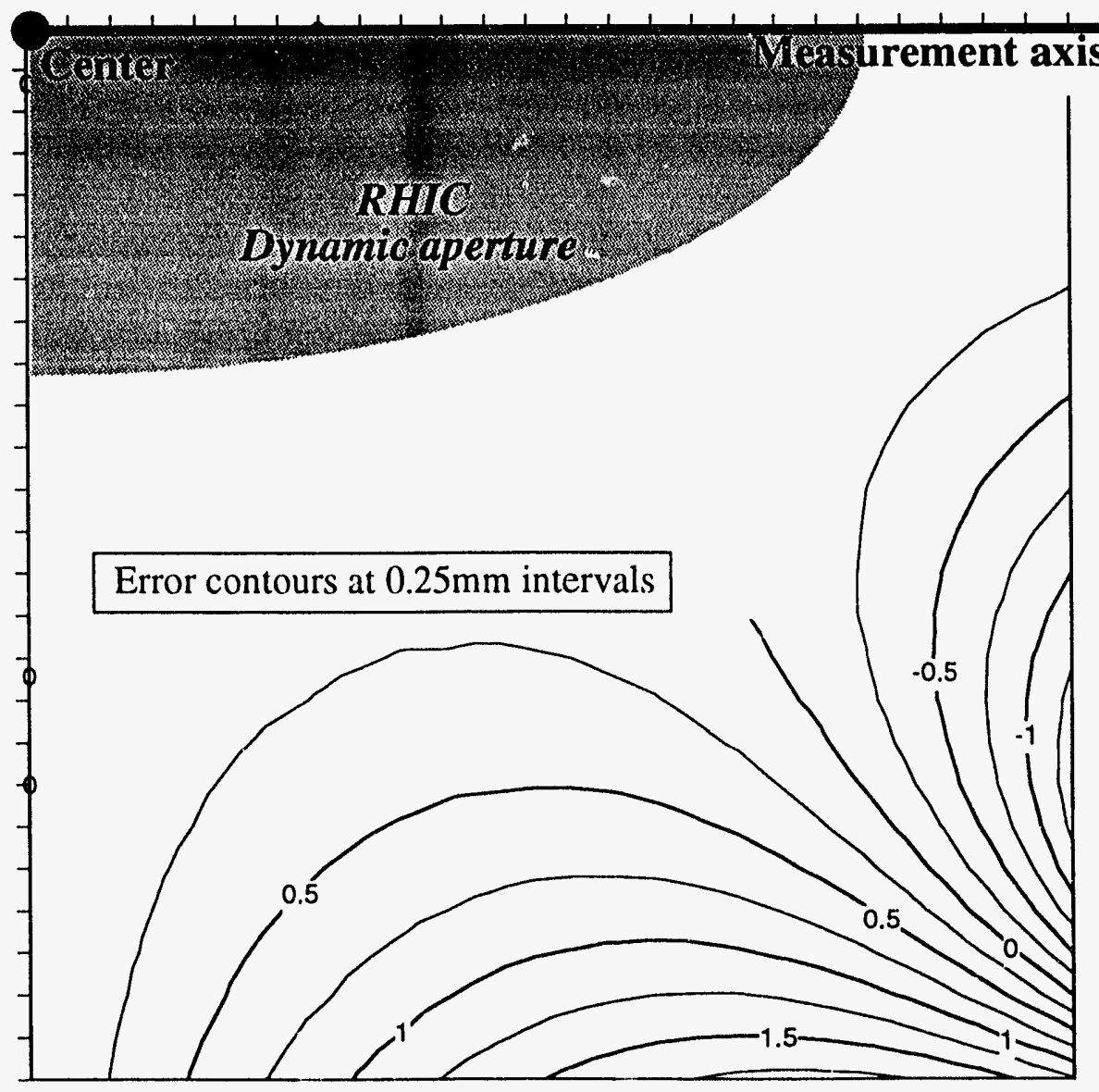

FIGURE 4. Error contours over a $2.5 \mathrm{~cm}$ square for $70^{\circ}$ electrodes 


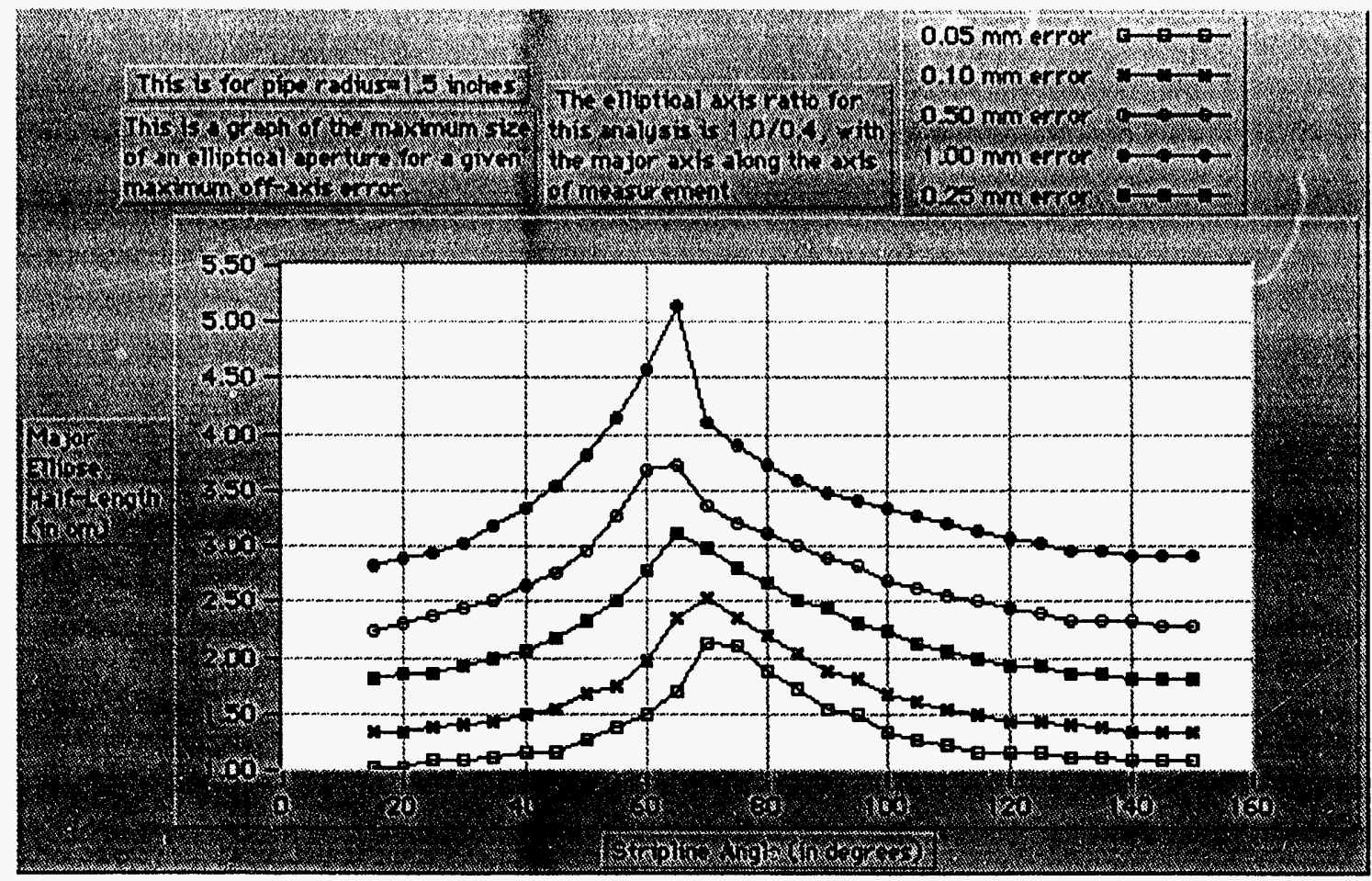

FIGURE 5. Maximum ellipse size vs. stripline angle for various errors

However, this will not give the correct $\mathrm{x}$ position. The resulting error is defined as the actual $\mathrm{x}$ position minus the position calculated from the on axis transfer function, or

$$
\text { error }=x-f\left(\frac{P_{A}}{P_{B}}\right)
$$

Writing Equation 4 in terms of $\mathrm{x}$ and $\mathrm{y}$ :

$$
\text { error }=x-f(g(x, y))
$$

The dynamic aperture at the position monitor location can be approximated by an ellipse with its major axis oriented along the measurement axis. The semimajor and semiminor axes are $2 \mathrm{~cm}$ and $0.8 \mathrm{~cm}$, respectively (The aspect ratio, 1:0.4, is taken from the square root of the ratio of the horizontal and vertical beta functions). Figure 4 shows a typical plot of error with the RHIC dynamic aperture superimposed. Figure 5 shows the maximum ellipse size that can fit within a certain error bound as a function of subtended angle.

\subsection{Coupling impedance}

The inductive part of the longitudinal coupling impedance due to the striplines alone (ignoring the effect of the cavity) can be calculated in the low frequency approximation with the following equation ${ }^{1}$

1. R. Schafer, IEEE Transactions on Nuclear Science, NS-32, No. 5, 1985. 


$$
\frac{Z_{L}(\omega)}{n}=2 Z\left(\frac{\phi}{2 \pi}\right)^{2} \frac{\omega_{0} l}{c}
$$

After substituting the parameters for the prototype RHIC position monitor:

$$
\begin{aligned}
& \omega_{0}=2 \pi \times 78 \mathrm{kHz} \text { is the angular revolution frequency } \\
& Z=50 \Omega \text { is the characteristic impedance of the stripline } \\
& \phi=80^{\circ} \text { is the subtended angle of one stripline } \\
& l=0.21 \mathrm{~m} \text { is the length of the stripline } \\
& c=3 \times 10^{8} \frac{\mathrm{m}}{\mathrm{s}^{2}} \text { is the speed of light }
\end{aligned}
$$

we obtain a longitudinal coupling impedance of $1.7 \times 10^{-3} \Omega$ per stripline pair, or approximately a total of $0.56 \Omega$ for the 332 pairs that each beam sees during one revolution. The effect of the position monitor cavity is being studied analytically and numerically.

\subsection{Electronics and System Considerations}

\subsection{Feature Set}

A position monitor module will provide two repositories for data: a circular buffer that is continuously updated called the closed orbit buffer, and a buffer that is filled on demand called the result buffer. Additionally, the modules will accept commands into a mode register and provide information on its current state to a status register. The closed orbit buffer should be large enough to store several seconds of data while the result buffer should store turn by turn data over a few synchrotron oscillations. Extra memory will also be allocated for transfer function lookup tables and signal processor executables. The following list describes the functions provided by a single position monitor module:

- closed orbit circular buffering

In this default mode of operation, closed orbit information is continuously updated at up to a $1 \mathrm{kHz}$ rate. This data is stored in a circular buffer. Acquisition is stopped only upon beam abort, special request, or during certain calibration sequences. At any time, the control system may read the current closed orbit information from the top of the closed orbit buffer.

- closed orbit series

At each occurrence of a particular synchronous event on the bunch clock, the module appends a new closed orbit data point to the result buffer. The repetition rate of this event should be less than $1 \mathrm{kHz}$. A series containing a certain number of data points is requested by writing to the mode register. The number of data points that remain to be acquired is available in the status register. 
- single bunch turn-by-turn series

By using a trigger derived from the bunch clock, the module acquires the position and intensity of a particular bunch for a certain number of consecutive turns. The acquisition is initiated by a single synchronous event on the bunch clock after which data are written into the result buffer at $78 \mathrm{kHz}$. The status register is then updated. If all of the modules are commanded to view the same bunch, that bunch's trajectory can then be reconstructed over thousands of turns.

- calibration

Some entries in a module's lookup table can be corrected with on-line calibration. A particular calibration sequence is initiated by a write into the mode register. Upon completion of the calibration sequence the lookup table is automatically modified, information about that calibration is stored in the result buffer, and the status register is updated.

\subsection{Analog receivers}

The development of receivers for the position monitor system is still in the early development phase. So far, a prototype following the block diagram in Figure 6 has been constructed and tested. The detector is linear over a a dynamic range of about $65 \mathrm{~dB}$ in each detected signal. The bandpass filters are centered at a $70 \mathrm{Mhz}$. This frequency was chosen as a compromise between signal maximization and reduction of the bunch spectrum dependence of the signal amplitude. The bandwidth of $>40 \mathrm{MHz}$ should allow suitable isolation between bunches. If self triggering is implemented, this isolation may have to be increased to allow for reliable triggering over the full dynamic range. Because the ratio of the two outputs is used to determine position, any noise and amplitude dependent delay from the limiter chain will eventually cancel. Any offsets will be observed on line by injecting a test pulse into a microstrip directional coupler close to the cryostat. This directional coupler will provide a precisely balanced pair of signals that emulate the beam induced pulses from striplines. Several other receiver designs are under construction for future evaluation.

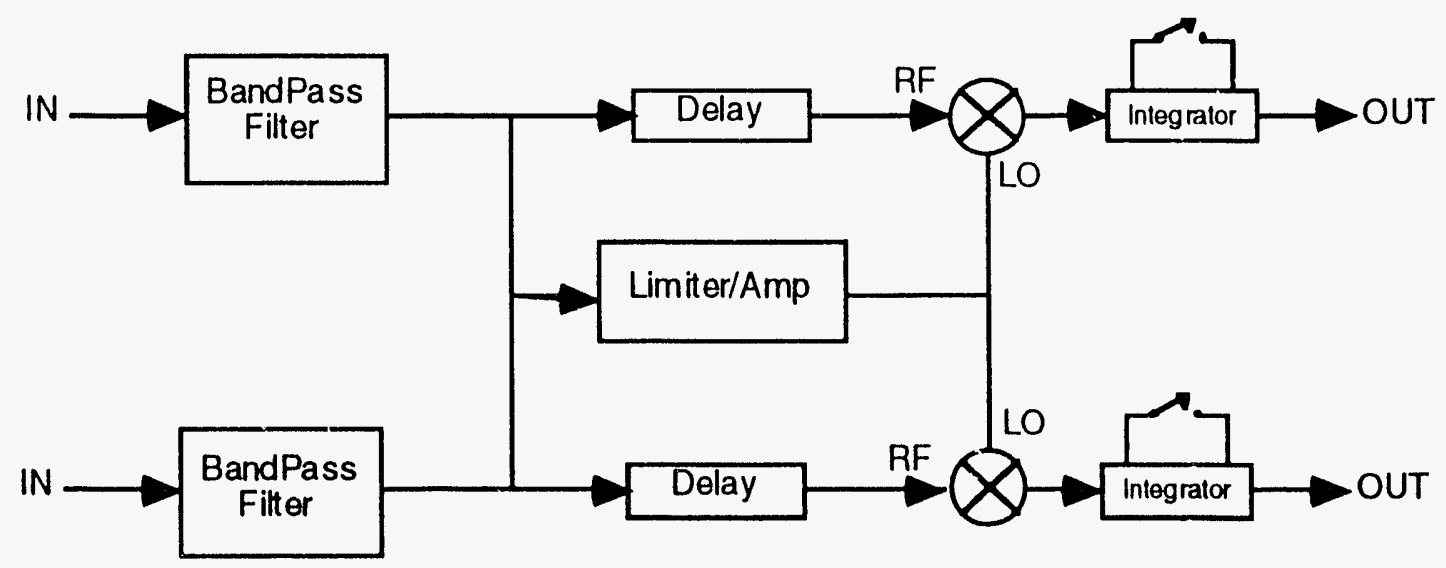

FIGURE 6. Block diagram of receiver prototype 


\subsection{Controls interface and timing}

The position monitor modules will be housed in a standard VXI chassis. All data for the position monitor system will be transferred to the VME based control system via a bus to bus interface. The buffers and registers mentioned in Section 4.1 will be memory mapped onto the VME address space to ease communication.

Three timing systems will synchronize the position monitor electronics: two bunch clocks (one per ring) and one event clock. The current design calls for the event clock to be based on the Fermilab Tevatron clock design. The two bunch clocks will operate with a carrier locked to the low level RF reference and each will carry a revolution event at $78 \mathrm{kHz}$. In addition, these clocks will carry any events used to synchronize systems to within one revolution period. An $8.9 \mathrm{MHz}$ clock will be locally derived from the bunch clock to facilitate bunch identification by individual position monitor modules.

\subsection{Acknowledgments}

The RHIC Beam Instrumentation Section members contributing to this design effort include: Marie Auduliene, Peter Cameron, Phil Cerniglia, Chris Degen, Vibha Mane, Tony Ryan, and Bob Sikora. 

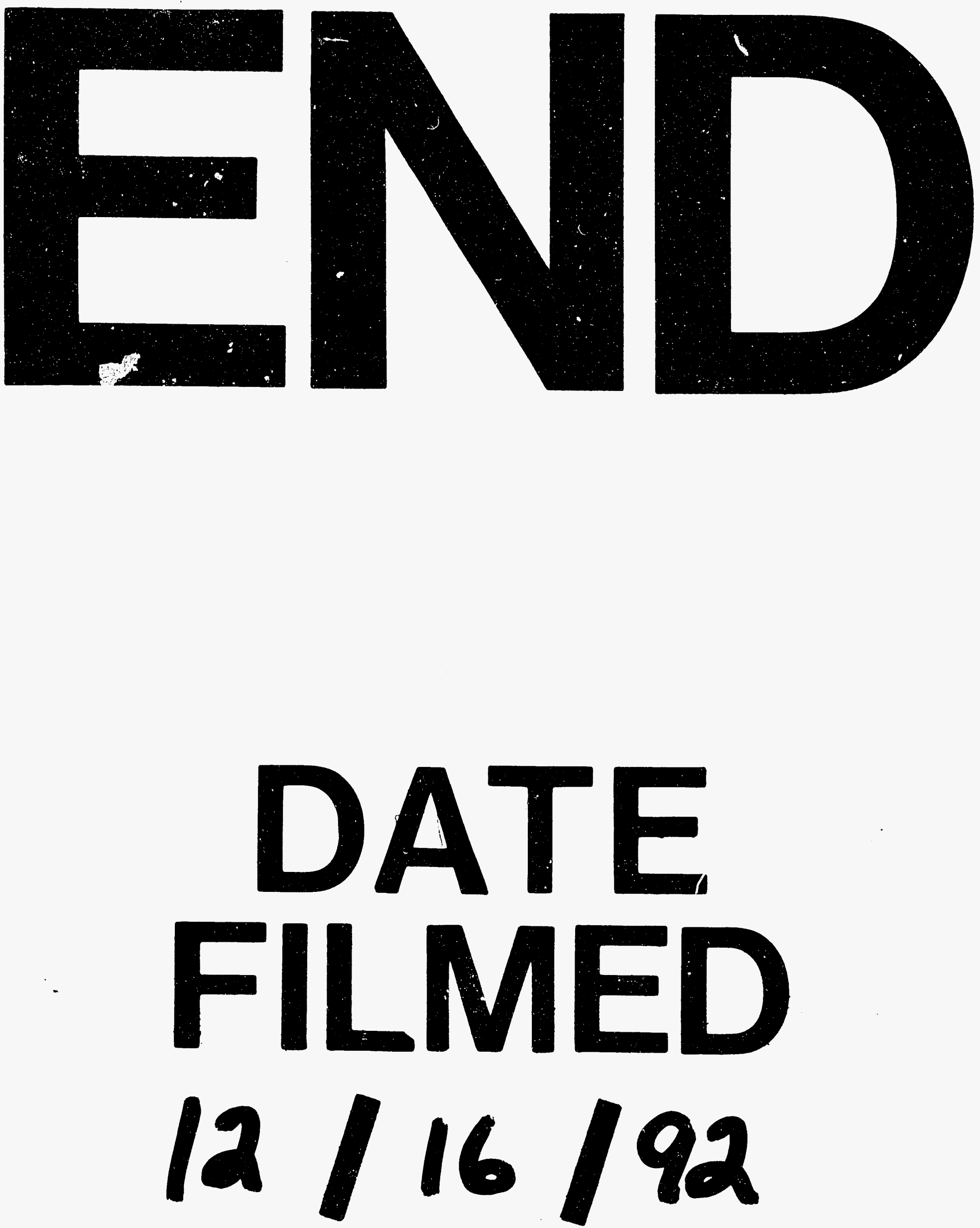
